\title{
Avaliação distribuição mitocondrial na maturação in vitro de oócitos bovinos de ovários com baixa e alta contagem de folículos antrais.
}

Letícia Zamberlan Pistillo ${ }^{1}$, Adriano Felipe Mendes ${ }^{1}$, Eder Pinatti ${ }^{2}$, Janaina Cristina Medeiros Fonseca ${ }^{1}$, Leandro Francisco Da Silva ${ }^{1}$, Mila Christeen Barbosa De Lima ${ }^{1}$, Wellington Ribeiro Martins ${ }^{1}$, Caliê Castilho ${ }^{1}$, Sheila Merlo Garcia Firetti ${ }^{1}$

${ }^{1}$ Universidade do Oeste Paulista - UNOESTE, ${ }^{2}$ Agência Paulista de Tecnologia dos Agronegócios - APTA. E-mail: lepe t@hotmail.com

\section{Resumo}

A Produção in vitro de embriões (PIVE) depende das etapas de maturação, fertilização e cultivo in vitro, sendo a maturação a etapa decisiva para o oócito atingir o estádio de mórula e blastocisto. Neste trabalho objetivou-se avaliar a qualidade de maturação in vitro, de acordo com a distribuição mitocondrial de oócitos provenientes de ovários de fêmeas de abatedouro, com baixa ou alta contagem de folículos antrais (CFA). Foram aspirados 243 ovários dos quais selecionou-se 334 oócitos perante de cinco repetições para cada grupo. Os ovários foram separados em dois grupos: 1- baixa CFA ( $n=19), 2$ - alta CFA $(n=53)$ e os oócitos recuperados corados com a sonda MitoTracker Red. Os dados obtidos foram analisados pelo teste não paramétrico exato de Fisher $(\alpha=5 \%)$ mediante o software Statistical Analysis System for Windows (SAS Inst., Inc., Cary, NC). Em relação a localização das mitocôndrias o presente trabalho apresentou diferenças $(p<0,001)$ entre oócitos imaturos e maturados, o que é desejável. Oócitos maturados apresentaram maior porcentagem de mitocôndrias dispersas no citoplasma e oócitos imaturos na periferia, independente do grupo experimental (baixa e alta CFA). Em oócitos imaturos, não houve diferença $(p=0,1542)$ na localização das mitocôndrias entre os grupos baixa e alta CFA. No entanto, o grupo baixa CFA, após a maturação, apresentou menor quantidade de oócitos com mitocôndrias na periferia em relação aos de alta CFA $(p=$ 0,0355), indicando melhor aquisição de competência desses oócitos para PIVE.

Palavras-chave: Bos indicus; Bos taurus; fêmeas; mitocôndria; produção in vitro de embriões.

Mitochondrial distribution evaluation in vitro maturation of ovarian bovine oocytes with low and high antral follicle count.

\begin{abstract}
In vitro embryo production (PIVE) depends on the stages of maturation, fertilization and in vitro cultivation, being the decisive stage for the oocyte to reach the morula and blastocyst stage. This study aimed to evaluate the quality of in vitro maturation, according to the mitochondrial distribution of oocytes from ovaries of slaughter females with low or high antral follicle count (CFA). A total of 243 ovaries were aspirated from which 334 oocytes were selected for five replicates for each group. The ovaries were separated into two groups: 1- low CFA $(n=19)$ 2- high CFA $(n=53)$ and stained with the Mito Traker Red probe. Data obtained were analyzed by Fisher's exact nonparametric test $(\alpha=5 \%)$ using the Statistical Analysis System for Windows software (SAS Inst., Inc., Cary, NC). Regarding the location of mitochondria, the present study showed significant differences $(p<0.001)$ between immature and mature oocytes, which is desirable. Mature oocytes had a higher percentage of dispersed mitochondria in the cytoplasm and immature oocytes in the periphery, regardless of the experimental group (low and high CFA). In immature oocytes, there was no difference $(p=0.1542)$ in the location of mitochondria between the low and high CFA groups. However, the oocytes of the low CFA group, after maturation, presented smaller number of oocytes with mitochondria in the periphery compared to the high CFA $(p=0.0355)$, indicating for this parameter a better competence of these oocytes for PIVE.
\end{abstract}

Keywords: Bos indicus; Bos taurus; females; mitochondria; in vitro embryo production. 


\section{Introdução}

A PIVE é uma biotécnica reprodutiva de extrema importância, que permite o contato entre o espermatozoide e o oócito mesmo fora do sistema reprodutivo da fêmea, de maneira que ainda possa ocorrer a formação de um novo indivíduo. $O$ processo envolve diversas etapas, das quais podemos citar a coleta dos oócitos, a maturação in vitro (MIV), a fecundação in vitro (FIV) e o cultivo in vitro (CIV) de embriões (GONÇALVES et al., 2008).

A MIV antecede todas as etapas realizadas na PIVE, e é nesse momento em que o oócito irá adquirir capacidade máxima para prosseguir nos próximos eventos, tais como FIV e CIV, expressando seu potencial de desenvolvimento após a fecundação (GOTTARDI, 2009).

Para atingir a metáfase II em seu estádio de blastocisto, o oócito sofre mudanças em seu citoplasma e núcleo, precisando ser maturado. Para isso acontecer, ele se encontra em diplóteno da prófase I e a remoção das células foliculares que estavam em contato com os oócitos, acaba por influenciar o processo de maturação nuclear, assim temos a meiose I que vai até a fase de metáfase II (CUNHA, 2014).

A finalidade principal das mitocôndrias é determinar a competência funcional dos oócitos (CUMMINS, 2004). Em oócitos imaturos as mitocôndrias irão aparecer distribuídas uniformemente na periferia do citoplasma, e após a maturação, elas irão aparecer como grupos maiores, e não permanecerão localizadas nas periferias, elas estarão principalmente na parte central do citoplasma o que não ocorrerá em oócitos considerados de má qualidade (STOJKOVIC et al., 2001).

Objetivo principal do trabalho foi verificar a influência da contagem de folículos antrais ovarianos sobre a qualidade da maturação in vitro de oócitos bovinos e avaliar o potencial de maturação, mediante redistribuição das mitocôndrias no citoplasma, de oócitos maturados in vitro.

\section{Metodologia}

Esse trabalho foi desenvolvido no laboratório da Universidade do Oeste Paulista, UNOESTE, Presidente Prudente - SP. Para sua realização foram utilizados 243 ovários de fêmeas bovinas zebuínas, provenientes do abatedouro
Bom Marte, localizado no município de Presidente Prudente - SP.

Foi realizado um pré-experimento para quantificação da média da contagem de folículos antrais, e possibilitar uma classificação dos grupos experimentais mais fidedigna. Foram contados os folículos antrais $\geq 3 \mathrm{~mm}$ visíveis da superfície de 200 ovários e de acordo com a distribuição normal dos dados classificou-se os grupos experimentais como G1: ovários que apresentaram menos que 19 folículos (baixa CFA = <19 folículos) e G2: ovário com mais que 53 folículos (alta CFA $=>53$ folículos).

Os ovários foram coletados no frigorifico Bom-Mart e transportados em garrafa térmica contendo solução salina a $0,9 \%$ de $\mathrm{NaCl}$, em temperatura de $37^{\circ} \mathrm{C}$. No laboratório os ovários, foram lavados com solução salina e mantidos em banho-maria a $37^{\circ} \mathrm{C}$ até a aspiração. Foram realizadas cinco repetições. Em cada repetição, antes da aspiração, os ovários foram separados de acordo com os grupos experimentais.

Para a aspiração folicular os ovários foram limpos com gaze estéril, e os oócitos aspirados com auxílio de seringas de $20 \mathrm{~mL}$ de 18G. Após a aspiração o líquido folicular foi transferido para tubos de ensaio mantidos em banho-maria a $37^{\circ} \mathrm{C}$ e decantado para separação dos oócitos. Após desprezar-se o sobrenadante, o sedimento foi depositado em placa de petri e os oócitos foram selecionados em lupa esteromicroscópica, (SMZ 745T, Nikon, Tókio, Japão), com aumento de 7,5x.

Os oócitos recuperados foram classificados de acordo com 0 aspecto morfológico dos COC's, em 5 grupos de qualidade, sendo Grau I = células do cumulus compacto presente, contendo mais de três camadas de células; Grau II = células do cumulus compacto parcialmente presente em volta do oócito ou rodeando completamente o oócito, com menos de três camadas celulares; Grau III = células do cumulus presente, com apenas uma camada de célula; Desnudo = ausência de camada de células do cumulus e Atrésicos = células do cumulus em regressão celular.

Para avaliação do potencial de membrana mitocondrial apenas os oócitos de grau I, II e III dos dois grupos foram selecionados. Metade dos oócitos foram avaliados antes da maturação e a outra metade submetidos à maturação in vitro. Para a maturação grupos de no máximo 30 oócitos, foram depositados em gotas de $90 \mu \mathrm{L}$ 
contendo meio de maturação, cobertas com óleo mineral (M8410, Sigma Co., St. Louis, EUA) e mantidos à temperatura de 38,5 ㄷ $\mathrm{Cem}$ atmosfera gasosa de $5 \%$ de $\mathrm{CO}_{2}$ em ar e com máxima umidade, por um período de 22 à 24 horas.

Para a avaliação da distribuição mitocondrial oócitos imaturos e maturados foram desnudados em vortex e corados com a sonda fluorescente MitoTracker Red CMXROs (M7512, Invitrogen, Eugene, EUA). Os oócitos foram incubados no escuro por 20 minutos em solução de PBS-PVP acrescido de $500 \mathrm{nM}$ de MitoTracker Red CMXROs, em temperatura de $37^{\circ} \mathrm{C}$ e posteriormente lavados em PBS. Feito o processo de coloração, foram transferidos para lâminas de vidro, recobertas com lamínulas e avaliados quanto à qualidade da maturação e quanto à predominância da distribuição das mitocôndrias no citoplasma (periférica ou dispersa) em microscópio equipado com epifluorescência (IX51, Olympus, Tókio, Japão), sob excitação de 579 nm e emissão de 599 nm.

Os resultados foram avaliados pelo programa computacional Statistical Analysis System for Windows (SAS Inst., Inc., Cary, NC) e o efeito da contagem de folículos antrais na redistribuição das mitocôndrias em oócitos antes e após maturação in vitro foi verificado por meio do teste exato de Fisher, ao nível de significância de $5 \%$.

\section{Resultados e Discussão}

No total foram aspirados 82 ovários de alta CFA e 161 ovários de baixa CFA, e selecionados 334 oócitos classificados como grau I, II e III.

A avaliação da distribuição mitocondrial foi realizada mediante coloração com a sonda fluorescente MitoTracker Red, este corante é vermelho-fluorescente e marca as mitocôndrias, sendo considerado uma importante ferramenta para avaliar a distribuição das mitocôndrias.

Segundo Dumollard et al. (2006), em ratos as mitocôndrias aparecem distribuídas uniformemente na periferia do citoplasma em oócitos imaturos. Romek et al. (2011), descreveram também que há o acúmulo de mitocôndrias na periferia antes da maturação e a formação de aglomerados de mitocôndrias localizadas no centro após a maturação em bovinos.

Entretanto, alguns trabalhos apresentaram resultados contraditórios sobre a localização e migração mitocondrial em bovinos. Machatkova et al. (2011), em seus estudos encontraram mitocôndrias distribuídas centralmente no citoplasma em oócitos imaturos em maior porcentagem em folículos médios ( $p<$ $0,05)$, sendo que os grupos experimentais eram oócitos imaturos e maturados, com folículos médios $(6-10 \mathrm{~mm})$ e pequenos $(2-5 \mathrm{~mm})$, no entanto, em oócitos maturados não houve diferença na frequência, com mitocôndrias distribuídas no centro tanto em folículos médios e pequenos.

Tarazona et al. (2006), descreveram uma distribuição periférica em oócitos maturados in vitro, onde observou o maior nível de fluorescência observado em oócitos maturados de $100 \%$ de atividade e seis níveis foram estimados, onde os grupos experimentais foram: (1) $0 \%$, (2) $1-15 \%$, (3) $16-30 \%$, (4) $31-50 \%$. (5) $51-75 \% 3$ (6) $76-100 \%$, em oócitos imaturos a atividade mitocondrial encontrada foi muito baixa e a distribuição foi difusa e insignificante.

O presente estudo apresentou diferenças significativas $(p<0,001)$ entre oócitos imaturos $e$ maturados em relação a localização das mitocôndrias. De acordo com a Tabela 1, pode-se observar que oócitos maturados apresentam maior porcentagem de mitocôndrias dispersas no citoplasma, e em oócitos imaturos estas estão localizadas na periferia, independente do grupo experimental (baixa e alta CFA).

Os resultados apresentados no Gráfico 1 demonstram que essa distribuição mitocondrial ocorre de maneira específica, observando-se uma intensa movimentação da periferia para o interior do citoplasma ao decorrer da maturação. 
Tabela 1. Localização mitocondrial de oócitos bovinos imaturos e maturados in vitro.

\begin{tabular}{|c|c|c|c|c|c|}
\hline & \multicolumn{5}{|c|}{ Localização das Mitocôndrias (\%) } \\
\hline & \multicolumn{2}{|c|}{ Disperso no citoplasma } & \multicolumn{2}{|c|}{ Periferia } & \multirow[t]{2}{*}{ Total $(\mathrm{N})$} \\
\hline & $(\%)$ & $(\mathrm{N})$ & $(\%)$ & $(\mathrm{N})$ & \\
\hline Oócitos imaturos & 4,85 & 8 & 95,15 & 157 & 165 \\
\hline Oócitos maturados & 94,67 & 160 & 5,33 & 9 & 169 \\
\hline$p<0,001$ & & & & & \\
\hline
\end{tabular}

Teste de Fisher, $\alpha=5 \%$

Gráfico 1. Distribuição mitocondrial em oócitos imaturos e maturados

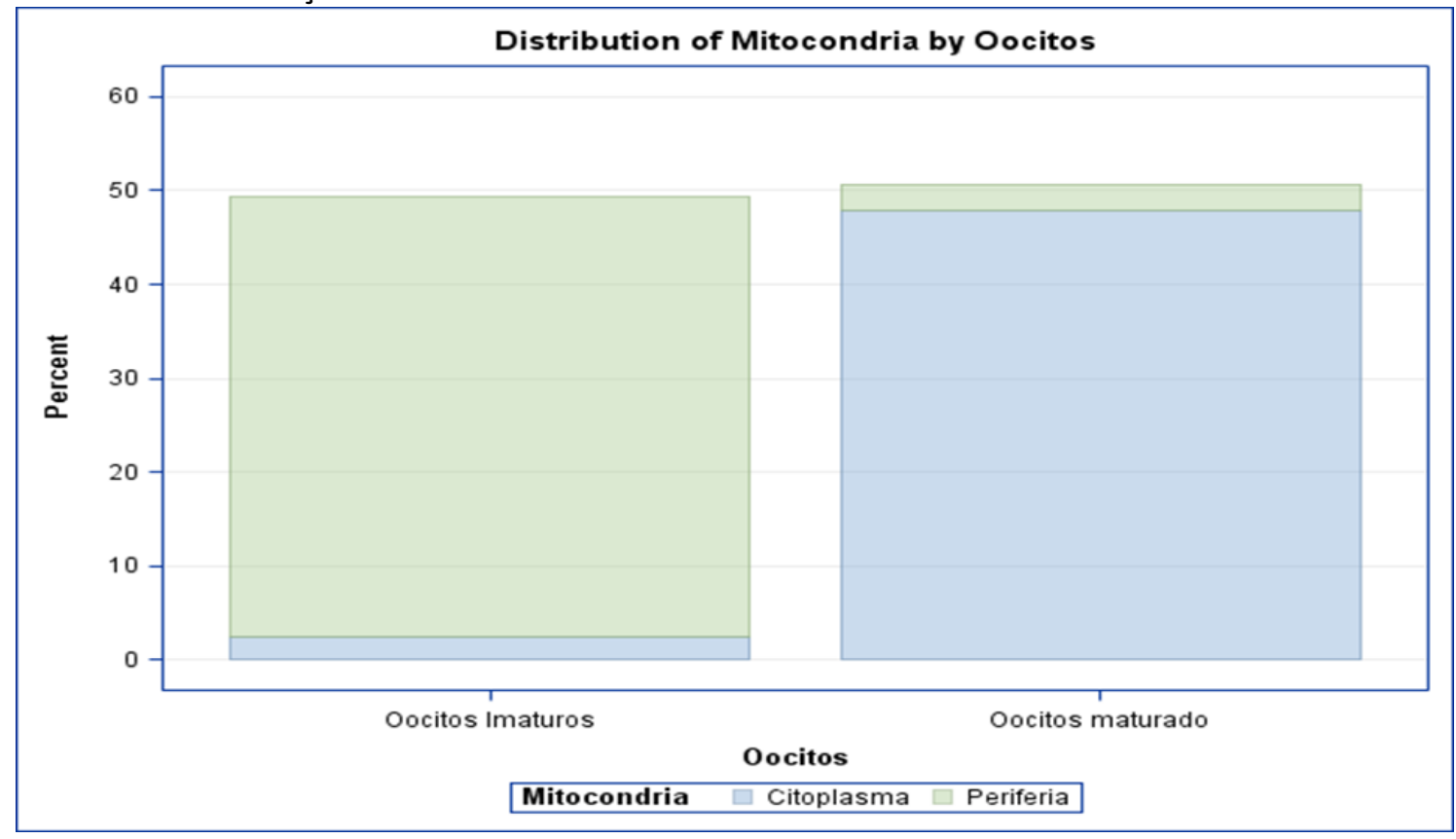

Brevini et al. (2005) também observaram esse comportamento em seu trabalho, onde regularam a maturação citoplasmática e a competência de desenvolvimento embrionário em oócitos suínos, com a presença (25\%) ou ausência (0\%) de líquido folicular suíno em meio de maturação.

Foi observado em seu estudo o conteúdo de ATP, a realocação ativa das mitocôndrias e a realocação de microtúbulos analisados em diferentes momentos durante a maturação in vitro, três distribuições mitocondriais foram identificadas: periférica, semiperiférica e difusa. Na coleta, $71 \%$ dos oócitos apresentaram distribuição periférica das mitocôndrias ativas, a realocação das mitocôndrias em direção ao centro do citoplasma iniciou-se por volta das 16 horas de maturação in vitro e foi concluída após 36 horas, nenhum deslocamento mitocondrial foi gravado após este tempo até o final do experimento (46 horas) (BREVINI et al., 2005).
A atividade e organização das mitocôndrias são escolhidas como um marcador facilmente detectável da compartimentalização do citoplasma, sua atividade e organização são características necessárias entre os diversos eventos envolvidos na maturação citoplasmática, sendo que sua principal função é gerar ATP, necessário para diversas funções (BREVINI et al., 2005).

No estudo de YU et al. (2010), os oócitos maturados apresentaram um alto percentual de mitocôndrias dispersas no citoplasma, sendo considerados de maior competência oocitária, já que a alta concentração de mitocôndrias está correlacionada com a produção de ATP e consequentemente com o desenvolvimento embrionário.

Quando há a perda ou diminuição da função mitocondrial ocorre de forma subsequente a baixa produção de ATP, este é considerado um dos fatores fundamentais que levam ao comprometimento da qualidade do oócito (BREVINI et al., 2005). Estudos apontam que $o$ desequilíbrio entre demanda e 
fornecimento de ATP devido ao comprometimento mitocondrial, pode resultar em falhas na maturação, parada da divisão celular e fragmentação (VAN BLERKOM, 2011).

Em relação à CFA não houve influência $(p=0,1542)$ na localização da mitocôndria em oócitos imaturos (Tabela 2). No entanto, no grupo baixa CFA após a maturação, observou-se menor quantidade de oócitos com mitocôndrias na periferia $(p=0,0355)$, indicando melhor potencial de maturação in vitro (Tabela 3 ).

Tabela 2. Localização mitocondrial de oócitos bovinos imaturos proveniente de ovários com Alta e Baixa CFA

\begin{tabular}{|c|c|c|c|c|c|}
\hline & \multicolumn{5}{|c|}{ Localização das Mitocôndrias (\%) } \\
\hline & \multicolumn{2}{|c|}{ Disperso no citoplasma } & \multicolumn{2}{|c|}{ Periferia } & \multirow[t]{2}{*}{ Total $(\mathrm{N})$} \\
\hline & (\%) & $(\mathrm{N})$ & (\%) & $(\mathrm{N})$ & \\
\hline Alta CFA & 7,59 & 6 & 92,41 & 73 & 79 \\
\hline Baixa CFA & 2,33 & 2 & 97,67 & 84 & 86 \\
\hline$p<0,1542$ & & & & & \\
\hline
\end{tabular}

Teste de Fisher, $\alpha=5 \%$

Tabela 3. Quantidade (N) e porcentagem (\%) de oócitos maturados de alta e baixa contagem de folículos antrais de acordo com a localização mitocondrial.

\begin{tabular}{cccccc}
\hline & \multicolumn{5}{c}{ Localização das Mitocôndrias (\%) } \\
\cline { 2 - 6 } & \multicolumn{6}{c}{ Disperso no citoplasma } & Periferia & Total (N) \\
\hline Alta CFA & $(\%)$ & $(\mathrm{N})$ & $(\%)$ & $(\mathrm{N})$ & \\
Baixa CFA & 90,91 & 80 & 9,09 & 8 & 88 \\
p $<0,0355$ & 98,77 & 80 & 1,23 & 1 & 81 \\
\hline
\end{tabular}

Teste de Fisher, $\alpha=5 \%$

A CFA é indicada como uma provável característica preditiva de fertilidade e tornou-se alvo de muitos estudos que têm procurado demonstrar que a quantidade de folículos antrais está associado positivamente a fertilidade em várias espécies, atuando sobre a produção de estradiol intrafolicular e também na expressão de alguns genes relacionados ao metabolismo do FSH (IRELAND et al., 2009).

Portanto, a mensuração e análise morfológica em estudos científicos podem auxiliar na determinação da capacidade reprodutiva (IRELAND et al., 2009). Os resultados encontrados neste trabalho em relação a CFA são semelhantes ao de Moraes (2016) que ao submeter vacas Bos indicus a IATF identificou não haver influência da CFA, porém notou uma tendência do grupo de baixa contagem a apresentar maior taxa de concepção em relação ao de alta CFA. Entretanto, cabe ressaltar que os estudos ainda são controversos, devendo-se associar a característica de CFA à outras ferramentas para a seleção de fêmeas doadoras. Morotti et al., (2015) ao avaliar a relação entre:
CFA, diâmetro de folículo e corpo lúteo em vacas Bos indicus também não encontrou nenhuma diferença em alta $(\geq 40)$ e baixa $(\leq 10)$ CFA.

Estes resultados vão de encontro a outros trabalhos, que encontraram inclusive maiores taxas de concepção em vacas com baixa CFA (RODRIGUES et al., 2013; MOROTTl et al., 2015).

Desta forma, os resultados encontrados neste estudo, indicam que há influência da CFA, na qualidade oocitária e na aquisição de competência para a maturação. No entanto, para poder relacionar esses dados à melhores índices reprodutivos, há necessidade de mais pesquisas que visem avaliar outras etapas do desenvolvimento embrionário.

\section{Conclusão}

Os oócitos do grupo baixa CFA após a maturação, mostrou melhor redistribuição mitocondrial, ou seja, menor quantidade de oócitos com as mitocôndrias na periferia em relação aos de alta CFA, desta forma sugerindo um maior potencial de maturação in vitro desses oócitos para a PIVE. 
Agradecimentos

Agradeço a Universidade do Oeste Paulista (UNOESTE).

\section{Referências}

BREVINI, T. A. L.; FRANCISCI, R. V. C.; GANDOLFI, F. Role of Adenosine Triphosphate, active mitochondria, and microtubules in the acquisition of developmental competence of parthenogenetically activated pig oocytes. Biology of Reproduction, v. 72, p. 1218-1223, 2005.

\section{https://doi.org/10.1095/biolreprod.104.038141}

CUMMINS, J. M. The role of mitochondria in the establixhment of oocyte functional competence. Eur J Obstet Gynecol Reprod Domest Anim, v. 115, n. 1, p. 23-29, 2004.

https://doi.org/10.1016/j.ejogrb.2004.01.011

CUNHA, M. C. R. V. A melatonina na maturação in vitro de oócitos bovinos. 2014. $100 \mathrm{f}$. Dissertação (Mestrado em Zootecnia e Engenharia de Alimentos) - Universidade de São Paulo, Pirassununga, 2014. Disponível em: http://www.teses.usp.br/teses/disponiveis/74/7 4131/tde-24092014-151935/pt-br.php. Acesso em: 11 abr. 2017.

DUMOLLARD, R.; DUCHEN, M.; SARDET, C. Calcium signals and mitochondria at fertilisation. Seminars in Cell e Developmental Biology, v. 17, p. 314-323, 2006.

https://doi.org/10.1016/j.semcdb.2006.02.009

GONÇALVES, P. B. D.; FIGUEREDO, J. R.; FREITAS, V. J. F. Biotécnicas aplicadas à reprodução animal. 2. ed. São Paulo: Varela, 2008.

GOTTARDI, F. P. Inibição da maturação nuclear pela butirolactona I durante o transporte de oócitos bovinos destinados à produção in vitro de embriões (PIV). 2009. 74 f. Dissertação (Mestrado em Reprodução Animal) Universidade Estadual Paulista, Jaboticabal, 2009. Disponível em:

https://repositorio.unesp.br/bitstream/handle/1 1449/98202/gottardi fp me jabo.pdf?sequence =1\&isAllowed=y. Acesso em: 15 abr. 2017.

IRELAND, J. J.; WARD, F.; JIMENEZ KRASSEL, F.; IRELAND, J. L. H.; SMITH, G. W.; LONERGAN, P.; EVANS, A. C. O. Follicle numbers are highly repeatable within individual animals but are inversely correlated with FSH concentrations and the proportion of good quality embryos after ovarian stimulation in cattle. Human Reproduction, v. 22, p. 1687 1695, 2009.

https://doi.org/10.1093/humrep/dem071

MACHATKOVA, M.; JESETA, M.; HULINSKA, P.; KNITLOVA, D.; NEMCOVA, L.; KANKA, J. Characteristics of Bovine Oocytes with Different Meiotic Competence in Terms of Their Mitochondrial Status and Expression of NuclearEncoded Factors. Reproduction in Domestic Animal, v. 47, p. 806-814, 2011. https://doi.org/10.1111/j.14390531.2011.01972.x

MORAES, F. L. Z. Influência da contagem de folículos antrais na taxa de concepção de vacas inseminadas em tempo fixo. 2016. $48 \mathrm{f}$. Dissertação (Mestrado em Saúde e Produção de Ruminantes) - Programa Associado entre Universidade Estadual de Londrina - UEL e Universidade Norte do Paraná - UNOPAR, Arapongas, 2016. Disponível em: https://repositorio.pgsskroton.com.br. Acesso em: 10 jun. 2019.

MOROTTI, F.; BARREIROS, T. R. R.; MACHADO, F. Z.; GONZÁLEZ, S. M.; MARINHO, L. S. R.; SENEDA, $M$. M. Is the number of antral follicles an interesting selection criterium for fertility in cattle. Animal Reprodution, v. 12, p. 479-486, 2015.

RODRIGUES, A. S.; OLIVEIRA, S. N.; FERRAZ, P. A.; LOIOLA, M. V. G.; COUTINHO, T. P.; SANTOS, M. M.; CHALHOULB, M. Antral follicle counts in Nellore females with different reproductive parameters and body condition score. Animal Reproduction, v. 10, p. 395, 2013.

ROMEK, M.; GAJDA, B.; ROLKA, M.; SMORAG, Z. Mitochondrial activity and morphology in developing porcine oocytes and pre-implantation non-cultured and cultured embryos. Reprod Dom Anim, v. 46, p. 471-480, 2011.

https://doi.org/10.1111/j.1439-

0531.2010.01691.x

STOJKOVIC, M. et al. Mitochondrial distribution and adenosine triphosphate contente of bovine oocytes before after in vitro maturation: correlaton with morphological criteria and developmental capacity after in vitro fertilization 
and culture. Biology of reproduction, Germânia, v. 64, p. 904-909, 2001. Disponível em: https://academic.oup.com/biolreprod/article/27 23403/Mitochondrial. Acesso em: 22 abr. 2017. https://doi.org/10.1095/biolreprod64.3.904

TARAZONA, A. M.; RODRIGUEZ, J. I.; RESTREPO, L. F.; OLIVERA-ANGEL, M. Mitochondrial activity, distribution and segregation in bovine oocytes and in embryos produced in vitro. Reproduction of domestic Animals, v. 41, p. 5-11, 2006. https://doi.org/10.1111/j.1439-

0531.2006.00615.x

VAN BLERKOM, J. Mitochondrial function in the human oocyte and embryo and their role in developmental competence. Mitochondrion, $\mathrm{v}$. 11, p. 797-813, 2011.

https://doi.org/10.1016/i.mito.2010.09.012

YU, Y.; DUMOLLARD, R.; ROSSBACH, A.; LAI, F. A.; SWANN, K. Redistribution of mitochondria leads to bursts of ATP production during spontaneous mouse oocyte maturation. Journal of Cellular Physiology, v. 224, p. 672-680, 2010.

https://doi.org/10.1002/jcp.22171 